\title{
Objectifs et moyens d'une politique de soutien d'étiage des rivières dans le Sud-Ouest français
}

\author{
Jean-Luc Redaud, Lucien Sormail, Agence de l'eau Adour-Garonne
}

Le développement actuel des surfaces irriguées dans le Sud-Ouest atlantique engendre des besoins en eau qui dépassent la disponibilité des rivières en période d'étiage si l'on veut sauvegarder un débit minimum nécessaire à l'équilibre écologique des rivières. Les sécheresses successives de 1989 et 1990 n'ont fait que confirmer ce décalage.

Ceci a conduit à la préparation dès 1988 d'un programme décennal de création de réserves en eau ambitieux, à double objectif : rétablir des débits d'étiage convenables sur les rivières, accompagner le développement des irrigations.

Le Comité de Bassin Adour-Garonne a défini les éléments de ce programme : 300 millions de $\mathrm{m}^{3}$ dans une quarantaine d'ouvrages à fonction de soutien d'étiage et 100 millions de $\mathrm{m}^{3}$ en retenues collinaires à vocation uniquement agricole.

Ce programme, en cours de révision, sera complété par un ensemble d'actions concourant à une meilleure gestion des réserves disponibles : économies d'eau, optimisation de la gestion tactique et stratégique des ouvrages, accords de déstockage avec les réserves hydroélectriques d'EDF.

Ce programme de barrages réservoirs a suscité des inquiétudes légitimes par les atteintes qu'il est susceptible d'apporter à l'environnement. Cet aspect du problème a fait l'objet de concertations approfondies sous l'égide d'une Commission " Amélioration des ressources en eau et écologie des rivières" dont les conclusions et recommandations ont été adoptées par le Comité de Bassin et diffusées auprès de tous les maîtres d'ouvrages.

Les objectifs et les moyens de cette politique sont développés ci-dessous.

\section{Un fort développement des consommations d'eau pour l'irrigation}

Compte tenu du climat du Sud-Ouest atlantique, des sécheresses d'été, l'irrigation est devenue une composante de la modernisation des exploitations agricoles dont le développement s'est accéléré. Avec l'intensification des productions, ce sont la régularisation et la diversification de celles-ci qui sont recherchées, bien que le maïs reste la culture irriguée dominante $(70 \%)$.

Les surfaces irriguées sont passées de 270000 ha en 1979 à 500000 ha en 1988 et représentent $10,3 \%$ de la SAU avec des points forts dans les départements du Tarn-etGaronne (19\%), du Lot-et-Garonne (21\%), des Landes (39\%).

Ces surfaces irriguées représentent en 1988, environ $50 \%$ des surfaces considérées comme potentiellement irri- gables sur le bassin ( $1000000 \mathrm{ha})$ avec les critères technico-économiques actuels.

De 1979 à 1988 le rythme moyen d'équipement a été de 25000 ha/an, mais il serait passé de 15000 ha/an au début des années « 80 » à plus de 40000 ha/an actuellement sous l'effet des sécheresses « 86 " puis 89 et « 90 ».

En ce qui concerne l'origine de l'eau utilisée pour l'irrigation, on peut considérer que sur les 500000 ha irrigués :

- $25 \%$ le sont à partir de retenues collinaires (soit 130000 ha);

$-15 \%$ le sont à partir de nappes profondes non reliées aux rivières ;

- $60 \%$ le sont à partir des cours d'eau et de nappes phréatiques (300 $000 \mathrm{ha})$.

La place prise par les retenues d'eau de type collinaire ( $25 \%$ soit près de $180 \mathrm{Mm}^{3}$ stockés dans plus de 8000 retenues de $10000 \mathrm{~m}^{3}$ à $2000000 \mathrm{~m}^{3}$ de capacité unitaire) constitue une caractéristique du Sud-Ouest.

Tous usages confondus (AEP, Industrie, Irrigation) le volume total consommé sur les eaux de surface serait de l'ordre actuellement de $450 \mathrm{Mm}^{3}$ qui correspond à une consommation moyenne de $45 \mathrm{~m}^{3} / \mathrm{s}$ sur 4 mois. Mais en réalité la part prépondérante de l'irrigation ( $85 \%$ ) dans cette consommation accentue le débit de pointe consommé sur 2 mois (juillet-août) que l'on peut évaluer à $90 \mathrm{~m}^{3} / \mathrm{s}$ actuellement.

Les prélèvements d'irrigation importants sur les cours d'eau et les nappes phréatiques concentrés sur juillet et août en période d'étiage, vont poser les problèmes majeurs d'aménagement et de gestion des eaux du bassin AdourGaronne.

\begin{tabular}{|l|c|c|c|}
\hline \multicolumn{4}{|c|}{ BASSIN ADOUR-GARONNE } \\
\begin{tabular}{|} 
Consommation eaux de surface du 1/7 au 31/10 \\
(rivières et nappes phréatiques)
\end{tabular} \\
\hline & 1979 & 1988 & " 2000 » \\
\hline AEP & $63 \mathrm{Mm}^{3}$ & $\begin{array}{c}69 \mathrm{Mm}^{3} \\
(15,5 \%)\end{array}$ & $\begin{array}{c}80 \mathrm{Mm}^{3} \\
(12,5 \%)\end{array}$ \\
\hline Industrie & $28 \mathrm{Mm}^{3}$ & $\begin{array}{c}19 \mathrm{Mm}^{3} \\
(4,5 \%)\end{array}$ & $\begin{array}{c}30 \mathrm{Mm}^{3} \\
(4,5 \%)\end{array}$ \\
\hline Irrigation & $185 \mathrm{Mm}^{3}$ & $\begin{array}{c}357 \mathrm{Mm}^{3} \\
(80 \%)\end{array}$ & $\begin{array}{c}545 \mathrm{Mm}^{3} \\
(83 \%)\end{array}$ \\
\hline Total & $276 \mathrm{Mm}^{3}$ & $\begin{array}{c}445 \mathrm{Mm}^{3} \\
(100 \%)\end{array}$ & $\begin{array}{c}655 \mathrm{Mm}^{3} \\
(100 \%)\end{array}$ \\
\hline
\end{tabular}




\section{Un objectif de débit minimum sur les rivières}

Les chroniques récentes de sécheresse (automne 1985, été 1986 , et surtout 1989 et 1990) se traduisant par des débits d'étiage très bas, ont permis de stigmatiser les conséquences d'une insuffisance de débit dans les rivières:

- abaissement du niveau d'eau perturbant le fonctionnement des prises d'eau, limitant l'espace vital de la faune piscicole et réduisant le potentiel récréatif d'une rivière ;

- dégradation spectaculaire de la qualité des eaux : réchauffement, eutrophisation ;

- aggravation des nuisances occasionnées par les usages de l'eau. Les rejets polluants sont moins bien digérés par la rivière (dilution et auto-épuration amoindries) et entrânent des risques d'insalubrité ainsi que des difficultés pour fabriquer de l'eau potable. Le fonctionnement des centrales hydroélectriques peut se traduire lorsque le débit de la rivière est trop faible par rapport aux débits turbinés, par des variations brutales du débit sortant à l'aval des installations, et même par son interruption momentanée.

Ces nuisances sont d'autant moins acceptées que le SudOuest veut préserver un espace et un cadre de vie accueillants.

La bonne santé des cours d'eau, la richesse des eaux vives, l'attrait des plans d'eau constituent des éléments forts de ce cadre de vie que les collectivités et les habitants veulent protéger.

En témoignage de cette préoccupation, les départements d'Adour-Garonne ont été parmi les premiers en France pour fixer des objectifs ambitieux de qualité des cours d'eau dans des cartes départementales d'objectifs de qualité.

C'est l'une des régions en France où la politique de restauration des rivières a été la plus active.

Enfin, la protection et la réintroduction des poissons grands migrateurs : saumon, alose, lamproie, font l'objet d'un programme ambitieux dans le Sud-Ouest.

Tous ces objectifs de restauration du milieu aquatique et ces efforts de lutte contre la pollution seraient remis en cause si les débits s'abaissaient trop souvent et gravement.

Ces considérations ont conduit les gestionnaires et aménageurs à définir un débit minimum à maintenir en tout point d'un cours d'eau et que ces consommations d'eau ne devraient pas amputer.

Dans les schémas d'aménagement des eaux étudiés ou réalisés en Adour-Garonne, cet objectif de débit a été appelé Débit Minimum Admissible (DMA).

Un tel concept peut être utilisé :

- soit pour limiter les consommations d'eau dans un bassin, afin de ne pas tomber trop souvent et trop fortement en dessous du DMA ;

- soit pour dimensionner et gérer des réserves de renforcement de débit d'étiage permettant de satisfaire de nouvelles consommations en respectant le DMA

Le concept d'un DMA étant acquis, la détermination de la valeur de ce débit doit tenir compte du régime hydrologique de la rivière, qu'il soit naturel ou influencé par d'anciens équipements de régularisation, et des usages particuliers de celle-ci, actuels et futurs.

Deux orientations principales peuvent être citées :

- Les débits de référence des cartes départementales d'objectifs de qualité : La procédure concertée d'établissement des " cartes d'objectifs de qualité » a permis de définir dans chaque département un cadre de référence pour les équipements industriels et collectifs de lutte contre la pollution des rivières. Les objectifs de qualité retenus sur chaque cours d'eau sont fondés sur le maintien d'un débit minimum de référence parallèlement à la diminution des pollutions à leurs sources.

Le débit d'étiage d'une rivière est un facteur fondamental du degré de qualité de ses eaux.

Généralement dans les dossiers "Objectifs de qualité ", les débits de référence retenus correspondent au minimum du débit moyen de 30 jours consécutifs que l'on observe une année sur cinq.

L'abaissement du débit en dessous du débit de référence des cartes d'objectifs de qualité entraîne une dégradation par rapport à l'objectif visé et peut annihiler l'effort consenti par les collectivités et les industriels pour réduire leur pollution.

Ces débits de référence deviennent alors de véritables objectifs de débit minimum.

- Les débits réservés de la loi "Pêche". Selon la loi, le débit réservé concerne la rivière à l'aval des ouvrages hydrauliques et doit être maintenu à tout instant dans la limite du débit arrivant à l'amont des installations. La loi « Pêche " de 1985 a introduit un concept simple pour fixer la valeur-plancher du débit réservé: le $1 / 10^{\mathrm{e}}$ du débit moyen interannuel ou module.

Le cadre réglementaire ainsi tracé, intéressant par sa simplicité de calcul et d'application, a le grand mérite de fournir la base d'une remise en ordre pour tous les équipements existants dont les contraintes de débits réservés apparaissaient hétérogènes et très faibles en général.

La valeur du $1 / 10^{\mathrm{e}}$ du module peut être envisagée comme valeur de DMA en vérifiant sa cohérence avec le régime particulier d'étiage de la rivière, le niveau de qualité de ses eaux, et les usages particuliers à chaque cours d'eau.

\section{Bilans besoins-ressources sur les rivières}

A l'échelle du bassin Adour-Garonne, le débit strictement consommé sur les eaux de surface, en période de pointe de consommation est de l'ordre de $90 \mathrm{~m}^{3} / \mathrm{s}$ et pourrait atteindre $130 \mathrm{~m}^{3} / \mathrm{s}$ à l'an « 2000 ».

Cette valeur est du même ordre de grandeur que la somme du débit d'étiage des grands cours d'eau du bassin en année sèche (référence 1 année/5):

$\begin{array}{llr}\text { - Charente } & : & 11 \mathrm{~m}^{3} / \mathrm{s} \\ \text { - Adour } & : & 17 \mathrm{~m}^{3} / \mathrm{s} \\ \text { - Dordogne } & : & 35 \mathrm{~m}^{3} / \mathrm{s} \\ \text { - Garonne } & : & 120 \mathrm{~m}^{3} / \mathrm{s} .\end{array}$


Le maintien de débits minima voisins des valeurs précédentes est donc compromis au moins une année sur cinq, si rien n'est fait pour compenser les consommations par des ouvrages de régularisation: réservoirs et ouvrages de transfert.

Un tel bilan permet de prendre une conscience globale de l'acuité de l'adaptation des ressources aux besoins.

Mais plus concrètement, l'estimation des besoins, le choix de D.M.A. et la comparaison aux débits d'étiage des rivières doit se faire sous-bassin par sous-bassin dans le cadre de schémas locaux d'aménagement des eaux.

Dans une région caractérisée par des saisons sèches et des baisses de débits correspondantes, les schémas réalisés font ressortir trois grandes familles de situation :

- De nombreuses rivières, ne prenant pas leur source en altitude et sans nappes alluviales importantes, ont des débits d'étiage naturels très faibles ou nuls: rivières du Lannemezan, affluents de l'Adour moyen, Lèze, Hers Mort, Sor, Dropt, etc...

Tout développement des usages le long de ces rivières impose alors de leur fournir en été une ressource artificielle répondant à la totalité des besoins et à l'objectif d'un débit minimum.

L'exemple le plus représentatif est celui des rivières du Lannemezan dont l'existence pendant plusieurs mois dépend totalement des réalimentations provenant du Canal de la Neste et de réservoirs de coteaux.

- D'autres rivières présentent des débits d'étiages appréciables (quelques $\mathrm{m}^{3} / \mathrm{s}$ ) résultant de conditions d'alimentation plus favorables (altitude, bassin versant, réserves souterraines d'alimentation...): Charente, Aveyron, Agout, Lot, Adour, Dronne, etc...

Mais l'importance des consommations, notamment agricoles, rend la rivière chroniquement inapte en période d'étiage à la satisfaction de tous les besoins, et surtout au respect de l'exigence du débit minimum. Les problèmes de pollution s'en trouvent exacerbés.

- Enfin, quelques grandes rivières présentent des débits d'étiage importants (quelques dizaines $\mathrm{de}^{3} / \mathrm{s}$ ): Garonne, Tarn, Ariège, Dordogne, Gave de Pau.

Le bilan des consommations sur ces grands axes est variable et conduit à des défaillances plus ou moins fréquentes par rapport au respect du D.M.A. Il atteint un seuil d'alerte pour la Garonne par exemple, qui accumule toutes les consommations sur ses affluents.

Ainsi, sur l'ensemble du réseau hydrographique d'Adour-Garonne, l'équilibre entre les débits d'étiage et les besoins, comprenant les objectifs de débit minimum, est menacé ou dégradé.

\section{Des sécheresses révélatrices}

Les sécheresses successives de 1989 et 1990 n'ont fait que confirmer le retard important accumulé depuis plusieurs années entre les besoins des irrigations et la disponibilité en eau. Les conséquences en ont été dramatiques dans tout le bassin Adour-Garonne. Elles se sont traduites :
- par un sacrifice généralisé d'une grande partie du réseau hydrographique (baisse des débits observés en deçà des débits réservés sur plus de $75 \%$ des stations hydrométriques du bassin, phénomène d'assèchement sur les petits cours d'eau avec mortalité piscicole);

- par des menaces graves à la sécurité de l'alimentation en eau potable de grandes unités de distribution d'un point de vue quantitatif (Montagne Noire) ou qualitatif (bloom d'algues en amont des prises d'eau de la basse vallée du Lot) et des risques de rupture pour des petits syndicats d'eau isolés ;

- par des pertes économiques importantes en dehors du monde agricole :

- surcoûts de production pour l'eau potable,

- réduction, voire arrêt, du fonctionnement des chaînes de production industrielles (usine de pâte à papier),

- arrêt de la navigation sur le Canal du Midi,

- mortalité arboricole en forêts,

- déstockages exceptionnels des réserves d'EDF.

Une forte mobilisation des services de l'administration, organisée en cellules départementales "sécheresse", a permis d'organiser au mieux la pénurie (bulletin de surveillance hydro-climatologique, définition de débits de crise, surveillance renforcée des rejets, définition de priorités dans les prélèvements).

Au plan agricole, elle s'est traduite dans tous les départements du bassin par l'instauration de tours d'eau, avec des interdictions d'irrigation plusieurs jours par semaine, par des appels aux économies d'eau et la lutte contre le gaspillage.

Ces mesures, mises en place dès juin 1989, furent reconduites dès le début de l'année 1990, parfois avec beaucoup de difficultés, notamment lorsqu'il s'agissait de partager la pénurie entre un département amont et un département aval.

L'instauration de mesures de régulation était en effet une innovation dans le Sud-Ouest où chacun, jusqu'à ces dernières années, croyait pouvoir puiser traditionnellement sans compter sur les rivières.

Malgré cela, les pertes agricoles furent très importantes en 1989 (de 30 à $40 \%$ sur les cultures non irriguées et 10 à $15 \%$ sur les cultures irriguées), soit environ 4 milliards de francs essentiellement en Midi-Pyrénées et PoitouCharentes (près de sept fois plus qu'en 1986).

En 1990, les pertes agricoles aggravées par un phénomène de canicule furent aussi importantes: elles purent cependant être limitées par un transfert important des soles de maïs au profit de cultures d'hiver non irriguées (céréales à paille et colza) et de tournesol. Dès le printemps 1990, les représentants de la profession agricole avaient en effet pu être alertés que la faiblesse des réserves naturelles (neige des montagnes, nappes) et du remplissage des barrages auguraient d'un été difficile.

Ces événements confirmaient le bien fondé du plan de développement des ressources en eau (P.D.R.E.) défini par le Comité de Bassin dès 1988, car il est évident que ces 


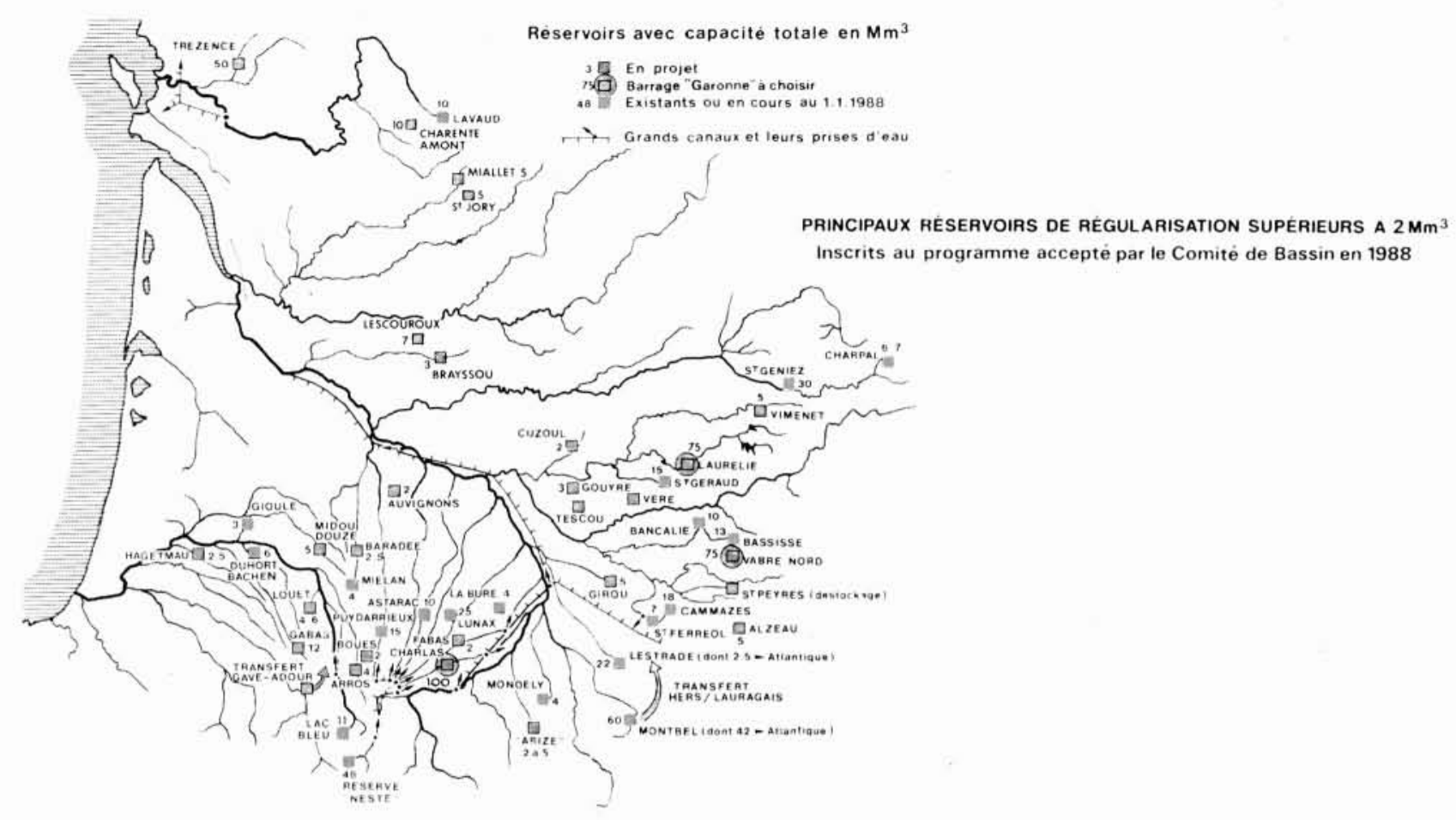

sécheresses ne sont que le révélateur d'un déséquilibre durable créé par un développement accéléré des surfaces irriguées.

\section{Le programme décennal de développement de ressour- ces en eau (PDRE)}

Pour rétablir des débits d'étiage convenables sur les rivières et pour accompagner le développement des irrigations, le Comité de Bassin Adour-Garonne a défini les éléments d'un programme ambitieux de construction de nouvelles réserves en eau : 300 millions de $\mathrm{m}^{3}$ dans une quarantaine d'ouvrages à fonction de soutien d'étiage et 100 millions de $\mathrm{m}^{3}$ en retenues collinaires à vocation uniquement agricole.

Ce programme initialement prévu sur 12 ans (1989-1998) se réalisera probablement sur 7 ans et son prolongement peut déboucher sur un objectif décennal de l'ordre de $600 \mathrm{Mm}^{3}$ de réserves. Elles s'ajoutent aux réserves existantes avant 1989: $400 \mathrm{Mm}^{3}$ dont 180 de retenues collinaires et 220 de soutien d'étiage.

Ce programme sera complété par un ensemble d'actions concourant à une meilleure gestion des réserves disponibles :

- économies d'eau (réhabilitation d'infrastructures anciennes - canaux, réseaux - conseil à l'utilisation rationnelle de l'irrigation à la parcelle sous contrainte...) ;

- optimisation de la gestion des lâchures en temps réel ; - accords de déstockage avec les réserves hydroélectriques d'EDF.;
- généralisation du comptage et de systèmes de tarification incitatifs.

L'investissement global de ce programme est de l'ordre de 3 milliards de francs et appelle un effort conjoint de l'Etat, des collectivités et de l'Agence de l'Eau. C'est un investissement financier très raisonnable si on le compare aux enjeux en péril et notamment aux pertes économiques constatées récemment.

Les ouvrages composant le PDRE se décomposent de la façon suivante :

a) 4 aménagements de transferts :

- Canal de la Neste : $14 \mathrm{~m}^{3} / \mathrm{s}$ et $110 \mathrm{Mm}^{3}$ pour les rivières du Lannemezan en période sèche,

- Canal de Saint-Martory : $8 \mathrm{~m}^{3} / \mathrm{s}$ pour la rive gauche de la Garonne en amont de Toulouse,

- Transfert Montbel-Lauragais : $12 \mathrm{Mm}^{3}$ pour le Lauragais,

- Transfert Gave-Adour : $15 \mathrm{Mm}^{3}$ pour l'Adour ;

b) 2000 petites retenues (de $20000 \mathrm{~m}^{3}$ à $1 \mathrm{Mm}^{3}$ ) totalisant $100 \mathrm{Mm}^{3}$;

c) 40 retenues de 1 à $2 \mathrm{Mm}^{3}$;

d) 15 retenues de 2 à $5 \mathrm{Mm}^{3}$;

e) 7 retenues de 5 à $10 \mathrm{Mm}^{3}$;

f) 7 retenues supérieures à $10 \mathrm{Mm}^{3}$.

Ce programme de barrages suscite des inquiétudes légitimes par les atteintes qu'il est susceptible d'apporter à l'environnement. Cet aspect du problème a fait l'objet de concertations approfondies sous l'égide d'une Commission "Amélioration de la ressource en eau et écologie des rivières ", pilotée par le Professeur Henri DECAMPS du CNRS et Monsieur l'Inspecteur Général EsTIENNE. 
Il convient en particulier que nous restions capables de montrer que l'effort de création de nouvelles réserves en eau concoura à maintenir dans les rivières les débits nécessaires à la préservation des autres activités (plan saumon, pêche, tourisme, navigation fluviale, eau potable et autres activités économiques).

Ceci implique un contrôle strict de développement des irrigations au prorata des nouvelles disponibilités de réserves en eau.

Malgré des progrès certains dans des zones comme celles maîtrisées par la CACG, des progrès importants restent à faire en ce domaine.

Le rapport de la Commission d'Etude dont l'avantpropos, joint en annexe, résume le contenu, a été largement diffusé auprès des maîtres d'ouvrages du bassin et constitue une ligne de référence pour les actions de l'Agence.

\section{Annexe}

Lors de la séance du Comité de Bassin du 8 juillet 1989, M. Jean FrançoIs-PONCET m'a demandé de bien vouloir présider la Commission d'Etude créée à son initiative et qui visait à évaluer l'impact du Programme de Développement de la Ressource en Eau sur l'équilibre écologique des rivières et d'élaborer des recommandations pour mieux harmoniser le choix, la conception et l'exploitation des aménagements prévus avec la protection des écosystèmes.

Une forte demande s'est aussitôt exprimée pour adhérer à ce groupe de réflexion et de proposition. De fait, près de 80 personnes se sont spontanément associées à notre démarche, et je me réjouis de l'esprit constructif dans lequel l'ensemble des participants de tous horizons, experts scientifiques, membres d'associations de protection de la nature, économistes, hydrobiologistes, universitaires, CEMAGREF..., ont travaillé sur un sujet difficile et passionnel.

La personnalité des présidents des trois groupes de travail qui ont été constitués et la qualité de leurs rapporteurs auront permis des approches de très haut niveau aboutissant à des recommandations pratiques :

- le groupe «piscicole » présidé par M. Delarue, Président de la Commission de Bassin, a ainsi examiné l'aptitude piscicole du milieu aquatique découlant des aménagements prévus, apprécié les risques liés à la construction de barrages-réservoirs, établi une liste de précautions à prendre, d'études préalables à faire lors de l'édification des barrages et posé des recommandations sur la gestion même des ouvrages ;

- le groupe " qualité des eaux ", présidé par M. Roger, Sénateur de Tarn-et-Garonne, s'est particulièrement attaché aux effets des stockages sur l'évolution de la qualité de l'eau, tant dans la retenue elle-même (risques d'eutrophisation) que sur les rivières réalimentées. Le groupe a souhaité que soit présenté un diagnostic de l'impact sur la qualité des eaux et leur évolution au niveau des études préalables et a suggéré des recommandations pour l'élaboration de protocoles de suivi de cette qualité des eaux sur de longues périodes;
- le groupe "vallée fluviale" présidé par M. BERNAD, Président de l'Association pour l'Aménagement de la Vallée du Lot, s'est intéressé aux vallées dominées par les futurs ouvrages et aux modifications éventuelles qu'ils sont susceptibles d'apporter tant à l'hydrosystème lui-même qu'aux activités humaines dont la rivière est le centre : sport, loisir, tourisme, etc... Le groupe a recommandé notamment que soit réalisé un cadre type d'impact de ces ouvrages sur la mise en valeur des vallées.

Le présent rapport synthétise ces contributions variées. Plus qu'un relevé de risques, il est un document de propositions concrètes qui peuvent être immédiatement suivies d'application: déjà, la suggestion de la création d'un Groupe de Travail Permanent a été reprise par le Comité de Bassin, qui a décidé de créer un "Comité Scientifique " de suivi de la réalisation du Programme de Développement de la Ressource en Eau.

Mais d'autres recommandations sont également porteuses d'avenir : par exemple celles qui expriment le souhait d'une étude globale d'environnement répondant au souci des uns et des autres de voir établir un diagnostic d'ensemble de l'impact du Programme; ou celles qui auront des répercussions sur certaines règles d'intervention de l'Agence de l'Eau, notamment dans les actions qui prévilégieront la garantie des soutiens d'étiages ou la maîtrise d'un développement raisonné des consommations.

L'essentiel de ces recommandations s'adresse d'abord aux maitres d'ouvrages et aux administrations de la police des eaux, véritables acteurs locaux chargés de conduire des procédures afférentes aux divers aménagements sur le terrain.

Ceci concerne les études d'impact dont le contenu devra prendre en charge plus largement les impacts sur l'ensemble des vallées dominées par les ouvrages et non les seuls impacts locaux.

Ceci concerne aussi une affectation claire des volumes d'eau réservés aux soutiens d'étiages et le contrôle de leur utilisation. En particulier les règlements d'eau des barrages devront préciser, avant tout début de réalisation, les objectifs de débit en rivière non seulement à l'aval immédiat des barrages, mais aussi en des points de référence plus éloignés pour lesquels il est prévu que la réserve en eau puisse assurer un débit minimum fixé par référence aux débits minimum admissibles des cartes d'objectifs de qualité. Ceci implique que les points de mesure soient installés à cet effet, et qu'un contrôle permanent de l'évolution des prélèvements d'eau soit mis en place afin de pouvoir veiller à ce que le développement des irrigations n'excède pas les volumes rendus disponibles à cet effet par les nouveaux stockages.

Des progrès importants restent à faire en ces domaines. La volonté des partenaires locaux de veiller à ces règles, est une condition primordiale du consensus et de la réussite collective d'une stratégie nécessaire de création de nouvelles réserves en eau dans nos régions.

Jacques EsTIENNE Ingénieur Général des Ponts et Chaussées 